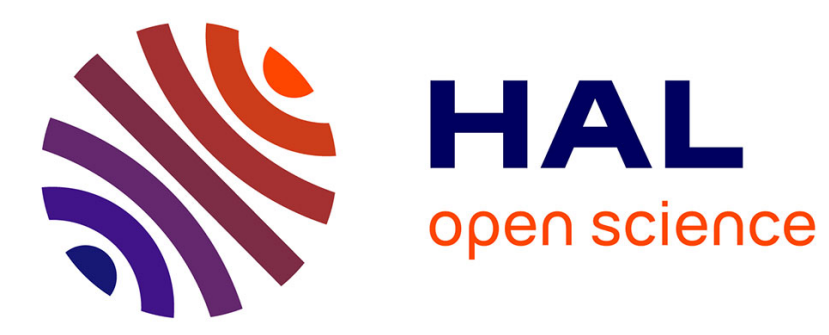

\title{
Position Estimation and Fall Detection using Visual Receding Horizon Estimation
}

\author{
Damien Brulin, Estelle Courtial, Guillaume Allibert
}

\section{To cite this version:}

Damien Brulin, Estelle Courtial, Guillaume Allibert. Position Estimation and Fall Detection using Visual Receding Horizon Estimation. 48th IEEE Conference on Decision and Control and 28th Chinese Control Conference, Dec 2009, Sanghai, China. hal-01994105

\section{HAL Id: hal-01994105 \\ https://hal.science/hal-01994105}

Submitted on 25 Jan 2019

HAL is a multi-disciplinary open access archive for the deposit and dissemination of scientific research documents, whether they are published or not. The documents may come from teaching and research institutions in France or abroad, or from public or private research centers.
L'archive ouverte pluridisciplinaire HAL, est destinée au dépôt et à la diffusion de documents scientifiques de niveau recherche, publiés ou non, émanant des établissements d'enseignement et de recherche français ou étrangers, des laboratoires publics ou privés. 


\title{
Position Estimation and Fall Detection using Visual Receding Horizon Estimation
}

\author{
Damien Brulin, Estelle Courtial and Guillaume Allibert
}

\begin{abstract}
The purpose of this paper is to estimate the position of a human in the image frame and to use this information to diagnose falls. A nonholonomic locomotion model describes the displacement of the human due to the similarities between human and nonholonomic mobile robot displacements. To estimate the human position in the world frame, the principle of Receding Horizon Estimation (RHE) is extended in the image plane. Indeed, this estimator is able to take into account an occlusion as a visual constraint. Residuals, errors between measured and estimated visual features, are generated to feed an alert dispositive. The latter will be used for the monitoring of an elderly person in a rest home. Thus the ground is assumed to be flat and a fixed perspective camera watches the scene. The simulations highlight the efficiency of the proposed approach, both without or with occlusions.

Index Terms-Receding Horizon Estimation, Human detection, Nonholonomic locomotion, Fall Diagnosis
\end{abstract}

\section{INTRODUCTION}

Human presence detection has become an important issue in our modern society. Indeed, we increasingly want to better manage our energy consumption, for instance by automating the lighting system. Furthermore, the life expectancy of humans has risen in the last decades and as a consequence, an improvement of the autonomy of the elderly turned out to be necessary. As most of the elderly prefer to stay in their own home, systems able to monitor the displacements and health have to be developed. Home automation has appeared to propose solutions. In [1], authors draw up an exhaustive list gathering more than 35 international projects of "smart houses". In all of these projects, a precise knowledge of the scene occupancy is required. So, the detection of human presence turn out to be important issues in home automation.

Several systems, from the state of the art, already offer solutions more or less reliable. We can cite Passive InfraRed detector (PIR), hyper-frequency sensor or thermopiles [2][3]. By misuse of language, these systems are frequently called "presence" detectors. In the majority of cases, they are simply movement detectors. Our work is in line with the CAPTHOM project of the Competitiveness Pole $S^{2} E^{2}$ (Sciences and System of Electric Energy). The objective of the CAPTHOM project is to really detect the presence of a human in an indoor environment by using a multi-sensors

D. Brulin is with Institut PRISME/MCDS UPRES EA 4229, ENSI Bourges, 88, bd. Lahitolle 18020 BOURGES Cedex, France damien.brulindensi-bourges. fr

E. Courtial and G. Allibert are with Institut PRISME/MCDS UPRES EA 4229, Polytech'Orléans, 8, rue Léonard de Vinci, 45072 Orléans Cedex 2, France estelle.courtialeuniv-orleans.fr, guillaume.alliberteuniv-orleans.fr system. The final objective consists in developing a system able to detect a situation of emergency, mainly a fall. The main danger for aged or handicapped persons is to fall in their apartment when they are alone. If we can quickly detect the emergency situation, we will be able to improve the alert dispositive. The first stage is then to estimate the position in the world frame from a visual measurement.

Among the different kinds of sensors, the camera appears to be well-adapted to give information about the presence of a human in a scene. As an image is a rich source of information, relevant data can be extracted by computer vision algorithms [5][6][9]. Within the CAPTHOM project, a computer vision algorithm has been developed for the human detection in an indoor environment [4]. The vision algorithm is able to detect a human and encloses him in a box. The coordinates of the middle point of each side of the bounding box are available. From this information in the image plane, we want to estimate the position of the human in the world frame. Many works about the position estimation of a moving object by visual information have been reported in computer vison literature. Unfortunately, visual measurements are very sensitive to noise and disturbances. Most of the time, an extended Kalman filter (EKF) is used [7][8] but, despite its prediction capability and its accuracy of estimation, the practical conditions limit its application. Furthermore, no constraints, like obstacle avoidance or image occlusion, can be taken into account The receding horizon estimation (RHE) can easily deal with constraints contrary to EKF [12]. Its principle is to formulate the estimation problem into an optimization problem over a past receding horizon [13].

The purpose of this paper is to extend the principle of the RHE to the position estimation in the image plane. The error between the visual feature measured thanks to the computer vision algorithm and the estimated visual features is to be minimized over the estimation horizon. The visual feature estimation is obtained by a global model combining the camera model and the human locomotion model. The latter is chosen as a nonholonomic model [15]. Image occlusions generally lead to aberrant position estimations. Here the image occlusion can be treated as a visual constraint, easily added to the optimization problem. The position estimation is then used in the generation of residuals for a diagnosis strategy.

The paper is organized as follows. In section II, the issue 
of human presence detection is introduced. Difficulties due to presence detection are underlined and the problem of fall detection is addressed. In section III, a global overview of the presence detection system is presented, introducing the camera and locomotion models. In section IV, the principle of the receding horizon estimation is briefly recalled. The proposed approach, called visual receding horizon estimation (VRHE), is then detailed. Finally, section V presents experimental results in two different configurations: a first one illustrating the method without constraints and a second one showing the efficiency of the method when occlusions occur. A description of our diagnostic method is also given.

\section{INHERENT DIFFICULTIES OF HUMAN PRESENCE DETECTION}

With regard to the current devices as PIR or ultrasound sensors, it is generally the movement which is detected and not the presence. If the movement is not sufficiently wide or in absence of movement, these devices become blind. Human presence detection sets out some difficulties.

\section{A. Problems linked to human presence detection}

The first problem which can appear is the detection of non-human targets. The system should be able to differentiate a detection brought out by the movement of an animal and the detection due to the presence of a human being. Two approaches can be envisaged to solve this differentiation problem. The first one is technological, simply by finding a sensor position that does not detect movement of small entities. The second one is rather software consisting in registering unmonitored areas of the scene or by defining threshold values.

Another issue of presence detection is the presence of several persons in the same room. Furthermore, a room can have more than one exit. So, one has to be able to manage the possibility that a person can enter in the room by one door and leave by a different door. Finally, because the presence of several persons can happen, the system must differentiate them and must adapt its behavior.

With computer vision algorithm, we can find solutions to deal with these problems. In [4], the authors have proposed a real time human detection based on visual information. The classification between human and non-human being is done with machine learning tools. Furthermore, each target is surrounded by a box which has its own identifying number. So we can also bypass the problem of multi-presence in a same environment and can track each target independently.

However, if the person falls, the computer vision algorithm is not able any more to decide if the target is a person or not. Automatic fall detection represents a difficult task to realize.

\section{B. The issue of fall detection}

The fall is responsible of $70 \%$ of the accidental death of the elderly. $30 \%$ of the elder fall at least once a year.
So there is clearly a need for automatic fall detection application. Different technologies have been investigated and can be classified in two groups : worn devices such as accelerometer or gyroscope and on the other hand, environment-based detection. For the second group, we can cite bed pressure sensors and video monitoring.

Worn devices are usually too invasive for the person. Within the CAPTHOM project, we focus on the use of vision sensor. The developed system should work with a simple camera, be easily implemented and cheap. Methods, based on computer vision, have been proposed in the literature to classify people's posture. Most of them use a 2D or 3D model of human body more or less detailed. In the majority of cases, a multi-camera approach is used to bypass the problem of occlusion [10]. Generally, vision methods use databases either with standing person images or with lying person images. Few methods combine the two kinds of databases because it would increase the computation time. It is then difficult to detect a fall. Moreover, it is important to differentiate a fall from a rapid body movement down. The purpose of this article is not to present a new vision algorithm but to extract the visual position information from the algorithm developed in [4] and to compare them with estimated positions to realize a diagnosis of the situation.

\section{OVERVIEW OF THE APPROACH}

The Figure 1. describes the presence detection system considered in our approach. The camera model and the human motion model used by the estimator are detailed below.

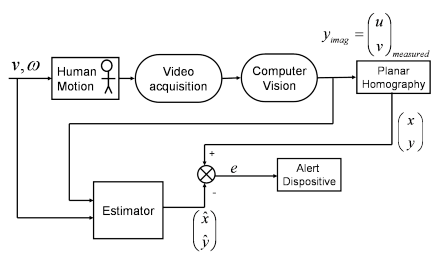

Fig. 1. Scheme of the VRHE

\section{A. The camera model}

As numerous methods using vision as source of information, one of the first steps consists in positioning and calibrating the camera so as to compute its model. We need to define a camera frame for the calibration and a world frame for the determination of the planar homography matrix (Figure 2). The latter will allow to determine the human coordinates in the world frame according to the coordinates in the image frame. Let's consider that the human is represented in the image plane by a point of interest, noted $P_{\text {int }}$. Thanks to the perspective camera model, we can write

$$
\begin{aligned}
& y_{\text {mod }}=\left(\begin{array}{c}
u_{\text {mod }} \\
v_{\text {mod }}
\end{array}\right)=\left(\begin{array}{c}
\alpha_{u} \frac{X_{c}}{Z_{c}}+u_{0} \\
\alpha_{v} \frac{Y_{c}}{Z_{c}}+v_{0}
\end{array}\right) \\
&\left(\begin{array}{c}
X_{c} \\
Y_{c} \\
Z_{c} \\
1
\end{array}\right)=M_{\text {trans }}\left(\begin{array}{c}
x_{h} \\
y_{h} \\
z_{h} \\
1
\end{array}\right)
\end{aligned}
$$




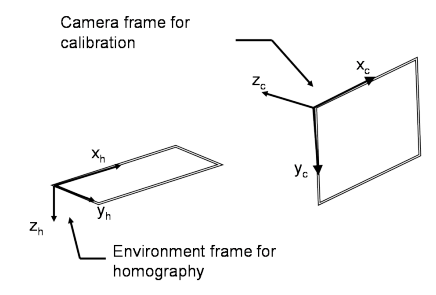

Fig. 2. Camera and Environment frames

where

- $\left(u_{\text {mod }}, v_{m o d}\right)^{T}$ are the coordinates in pixels of $P_{\text {int }}$ in the image frame;

- $\left(X_{c}, Y_{c}, Z_{c}\right)^{T}$ are the coordinates of $P_{\text {int }}$ in the camera frame;

- $\alpha_{u}, \alpha_{v}, u_{0}, v_{0}$ are the camera intrinsic parameters;

- $\left(x_{h}, y_{h}, z_{h}\right)^{T}$ are the coordinates of the human in the environment frame.

Equation (2) is the relationship between the camera frame and the world frame. The details of this matrix will be discussed in the experimental section. We assume that the person walks on a flat ground ( $z_{h}$ is constant).

\section{B. Human locomotion model}

In the Figure 1, the block called human motion corresponds to the locomotion model. In order to estimate the position of a person, the knowledge of its displacement is necessary. However, modeling and describing precisely the movement of a human is not an easy task because it does not follow precise rules. A first approach can consist in simulating the movement by a simple integrator, leading after a first order discretization to a linear motion model. Another method is to express the motion of a human by a nonholonomic model [15]. This choice is motivated by the fact that the behavior of the human is very similar to the behavior of a nonholonomic mobile robot. Humans do not walk sideways : some constraints of different natures (anatomical, mechanical) may restrict the human locomotion trajectories. These constraints can be expressed under the so called "rolling without slipping" constraint. The human locomotion model is then written as :

$$
\dot{p_{h}}(t)=f\left(p_{h}(t), u(t)\right)=\left(\begin{array}{c}
\cos \theta_{h} \\
\sin \theta_{h} \\
0
\end{array}\right) v+\left(\begin{array}{l}
0 \\
0 \\
1
\end{array}\right) \omega
$$

where $p_{h}=\left(x_{h}, y_{h}, \theta_{h}\right)^{T}$ is the human posture with $\theta_{h}=\arctan \frac{\dot{y}_{h}}{\dot{x_{h}}}$ and the control inputs $u=(v, \omega)^{T}$ are respectively the linear and angular velocities.

\section{The global model}

The global model is composed of the camera model (1), the transition matrix (2) and the human motion model (3). We can then resume this global model by (4) :

$$
\left\{\begin{array}{l}
\dot{p_{h}}(t)=f\left(p_{h}(t), u(t)\right) \\
y_{\text {mod }}(t)=h \circ M_{\text {trans }} \circ g\left(p_{h}(t)\right)=H\left(p_{h}(t)\right)
\end{array}\right.
$$

where $p_{h}(t)$ and $y_{\text {mod }}(t)$ are respectively the human position in the environment frame and the image frame. $h$ is the camera model, $M_{\text {trans }}$ is the transition matrix and $g$ allows to transform the position $\left(x_{h}, y_{h}, z_{h}, 1\right)^{T}$ to the posture vector $\left(x_{h}, y_{h}, \theta_{h}\right)^{T}$.

This global model will be used to estimate the human position from the knowledge of visual information.

\section{Visual Receding Horizon Estimation}

\section{A. Receding Horizon Estimation (RHE)}

The estimation of the position and orientation of a moving object has been largely investigated in the literature over the last few years. The estimation of the pose of the target is often required in position-based visual servoing approaches. Kalman filtering, especially the EKF, offers a satisfactory rejection of disturbance or noise and an accurate estimation. In [11], the authors proposed an adaptative version of EKF for visual applications. However, the EKF may encounter difficulties for practical implementations when state constraints have to be handled and when the process is highly nonlinear [12]. In order to overcome these problems, a RHE strategy can be used. The state estimation is formulated as solving on-line a nonlinear optimization problem. Constraints can easily be added to the optimization problem [13].

We propose to extend the receding horizon estimation concept to visual estimation.

\section{B. Visual Receding Horizon Estimation (VRHE)}

The estimation problem of the human position is then written as a nonlinear optimization problem in the image plane over a past receding horizon $N_{e}$. The difference between the measured features in the image plane denoted $y_{\text {imag }}$ and the estimated features denoted $y_{\text {mod }}$ defines the cost function $J$. The estimated features are obtained thanks to the global model combining the human motion model and the camera one. The cost function $J$ is to be minimized with respect to the human position $p$ at time $k-N_{e}$. The position estimation at the current time $k$ is then computed thanks to the human motion model and the initial state estimate $p_{k-N_{e}}$.

At each sampling time, the past finite estimation horizon moves one step forward and the procedure is repeated to ensure the robustness of the approach in regard to disturbances and model mismatches.

The cost function can be written in discrete-time as:

$J(p)=\sum_{j=k-N_{e}}^{k}\left[y_{\text {imag }}(j)-y_{\text {mod }}(j)\right]^{T} Q\left[y_{\text {imag }}(j)-y_{\text {mod }}(j)\right]$

$\mathrm{Q}$ is a symmetric definite positive matrix. The mathematical formulation of the Visual RHE is then given by:

$$
\min _{p_{k-N_{e}}} J(p)
$$

subject to: 


$$
\left\{\begin{array}{l}
p_{h}(k+1)=F\left(p_{h}(k), u(k)\right) \\
y_{\text {mod }}(k)=H\left(p_{h}(k)\right)
\end{array}\right.
$$

The nonlinear equation (7) describes the dynamics of the global model (4) in discrete time.

One of the main advantages of VRHE is the capability to explicitly take into account constraints contrary to EKF.

\section{Simulation AND Results}

The feasibility and the performance of the proposed visual estimation method have been experimentally tested using a single perspective camera. Two different cases are considered. First of all, videos where there are no occlusion of the human are used to illustrate the feasibility of our method. The second aims at studying the capability of the proposed method to deal with large path variations due to occlusion. For each case, several videos have been tested to vary movements in the observed scene. No occlusion model is used. The occlusion is treated as a visual constraint in the estimation procedure.

\section{A. The computer vision algorithm}

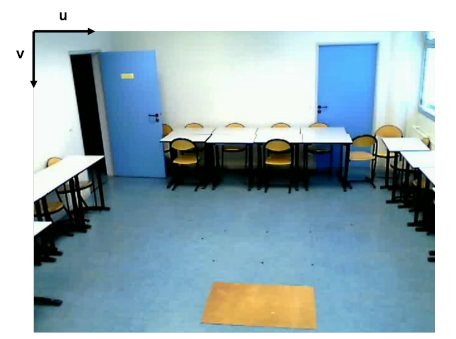

Fig. 3. Camera's view of the scene and image frame

The camera has been calibrated by using a least square method. The resolution of the camera is $640 \mathrm{x} 480$ pixels but in the display result of the computer vision algorithm, the image size is reduced to $320 \times 240$ pixels. The sampling period used is the minimum time allowed by the camera frame rate, $T_{e}=0.07 \mathrm{~s}$. The camera was placed at a height of $1.98 \mathrm{~m}$. The scene viewed by the camera is illustrated in Figure 3.

The change from the environment frame to the image frame is given by the matrix $M_{i n t r}$ obtained with the calibration of the camera. Moreover, a transformation matrix $M_{h o m}$ is required for the change from the image to the environment frame. In [14], the authors use a planar homography matrix to compute the position of a flame front on the ground in the world frame from its position in the image. To compute this homography matrix we used a frame, which can be seen in Figure 3., measuring $0.92 \times 0.59 \mathrm{~m}$. Because we used two different environment frames (Figure 2.), a third matrix $M_{\text {trans }}$ is needed to compute the transition between both frames.

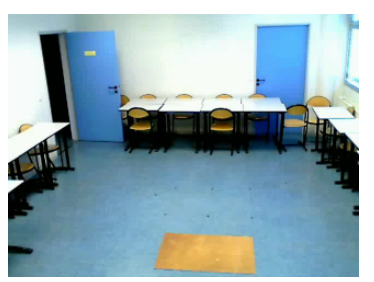

(a)

(c)

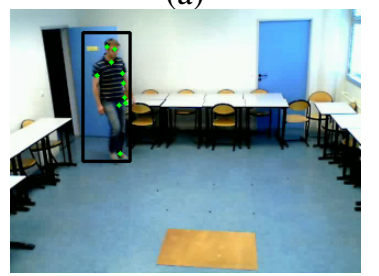

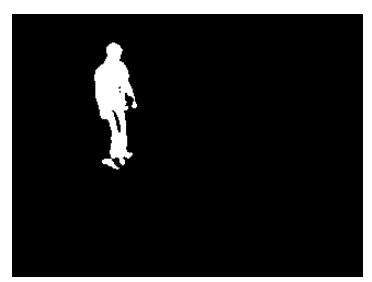

(b)

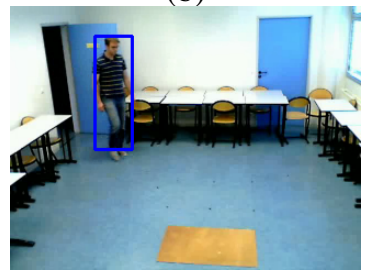

(d)
Fig. 4. Representation of the different steps of the computer vision algorithm

In brief, the three different transition matrices are :

$$
\begin{aligned}
M_{\text {intr }} & =\left(\begin{array}{ccc}
328.17 & 0 & 170.88 \\
0 & -327.89 & 103.51 \\
0 & 0 & 1
\end{array}\right) \\
M_{\text {hom }} & =\left(\begin{array}{cccc}
0.0065 & 0.0035 & -1.4892 \\
-0.0003 & 0.0175 & -3.7361 \\
0.0003 & 0.0033 & -0.4043
\end{array}\right) \\
M_{\text {trans }} & =\left(\begin{array}{cccc}
1 & 0 & 0 & -1.02 \\
0 & 0 & 1 & 1.98 \\
0 & -1 & 0 & 5.11 \\
0 & 0 & 0 & 1
\end{array}\right)
\end{aligned}
$$

where $M_{\text {intr }}, M_{\text {hom }}$ and $M_{\text {trans }}$ are respectively the intrinsic parameter matrix, the homography matrix and the transition matrix between the environment frame of homography and the environment frame of calibration.

The principle of the computer vision algorithm [4] is depicted in the Figure 4. A preliminary step of the program is to take some pictures of a clear scene in order to have a fixed background (a). Changes are detected by computing the Mahalanobis' distance between pixels of the current image and the background model (b), composed of the mean of the three RGB components and of the co-occurrence matrix. This first step is done in order to reduce the search space of the classifier. Then, the preceding detected objects are observed by using a tracking of point of interest (c). The final step consists in determining the nature of the tracked object (d). Authors built a cascade of boosted classifiers based on Haarlike filters and on a boosting method to discriminate human and non human entities. The detected person is enclosed in a box. The human position, in the image plane, is then given by the coordinates of the middle point of the box's bottom side :

$$
y_{\text {imag }}=\left(\begin{array}{l}
u_{\text {mes }} \\
v_{\text {mes }}
\end{array}\right)
$$




\section{B. Computation of the locomotion model}

A numerical computation is performed to obtain the motion velocity profiles from the visual measurements. The linear and angular velocities are calculated at each step by :

$$
\left\{\begin{array}{l}
v_{x}(j)=\frac{x_{h}(j)-x_{h}(j-1)}{T_{e}} \\
v_{y}(j)=\frac{y_{h}(j)-y_{h}(j-1)}{T_{e}} \\
v(j)=\sqrt{v_{x}^{2}(j)+v_{y}^{2}(j)} \\
\theta_{h}(j)=\arctan \left(\frac{v_{y}(j+1)}{v_{x}(j+1)}\right) \\
\omega(j)=\frac{\theta_{h}(j)-\theta_{h}(j-1)}{T_{e}}
\end{array}\right.
$$

With a first order discretization, the nonholonomic model of human motion can be written as :

$$
\left\{\begin{array}{l}
x_{h}(j+1)=x_{h}(j)+T_{e} \cos \left(\theta_{h}(j)\right) v(j) \\
y_{h}(j+1)=y_{h}(j)+T_{e} \sin \left(\theta_{h}(j)\right) v(j) \\
\theta_{h}(j+1)=\theta_{h}(j)+T_{e} \omega(j)
\end{array}\right.
$$

Finally, the position estimation of the human is performed by solving the nonlinear optimization problem (6) subject to equations (7) and (11).

\section{Simulations without any occlusion}

For all experimentations, the size of the estimation horizon is fixed to $5\left(N_{e}=5\right)$, the matrix $Q$ is the identity matrix. The VRHE algorithm has been implemented in Matlab software and the computer vision algorithm in $\mathrm{C}++$.

In this case study, scenarios have been realized in a room without any occlusion. The aim was to verify the feasibility of the method with a simple case. The Figures 5 and 6 illustrate respectively the estimation of the position with VRHE according to $x$ and $u$.

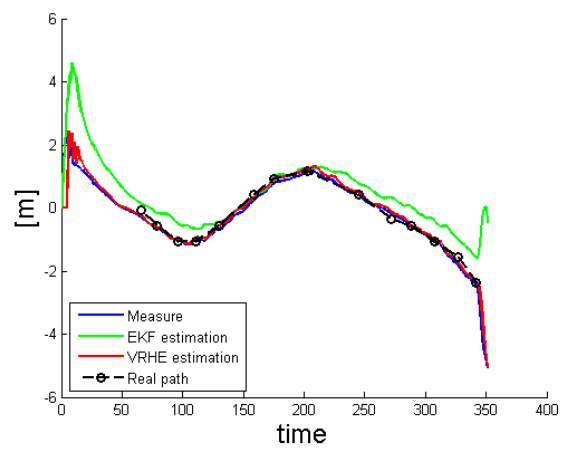

Fig. 5. Time history of the measured trajectory and the estimated trajectory according to $\mathrm{x}$

The VRHE begins to run as soon as the program has reached the estimation horizon $N_{e}$ and has sufficient information that is why the first five points are at zero. As we can see, the estimate trajectory is closed to the real path in both directions. The estimation obtained with VRHE is similar to the estimation of the EKF. It must be noticed that the velocities $v_{x}$ and $v_{y}$ are filtered because there are computed from noisy image data. Same results have been obtained with several videos and have proved the feasibility of the proposed approach.

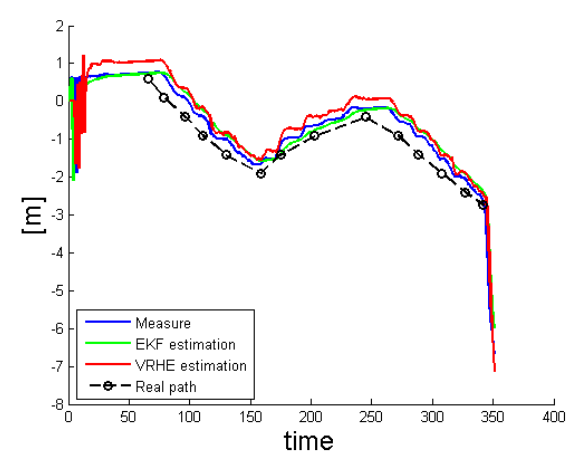

Fig. 6. Time history of the measured trajectory and the estimated trajectory according to $\mathrm{y}$

\section{Simulations with an occlusion}

If the person walks behind an obstacle, a problem of occlusion will appear and the size of the box determined by the computer vision algorithm will suddenly change, leading to deviant measures. The Figure 7 illustrates the problem of occlusion. (a) depicts the scene viewed by the camera. Before the person walks behind the obstacle, the box correctly includes the person (b). When the person is partially masked by the obstacle (c), we clearly see that the box is two times smaller than the previous one. Once the person is no longer hidden by the obstacle, the box recovers its original size (d). To deal with an occlusion, two approaches

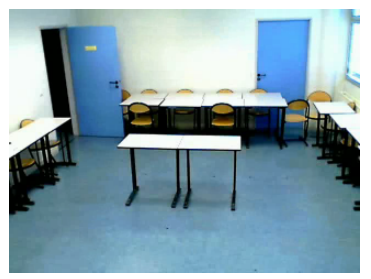

(a)

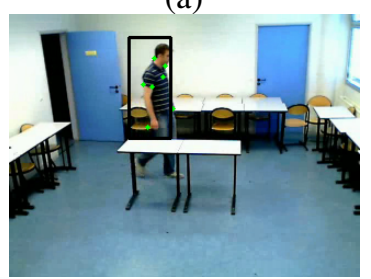

(c)

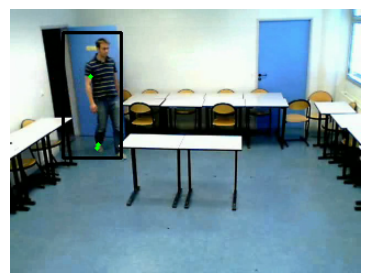

(b)

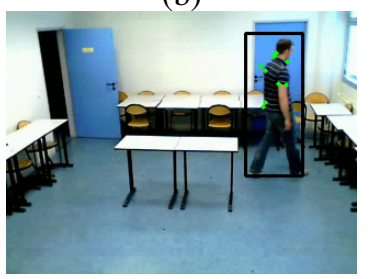

(d)
Fig. 7. Representation of the problem of occlusion

exist. A constraint on the admissible displacement based on the past movements can be taken into consideration in the nonholonomic model. Another strategy is to determine, by image processing, obstacle dimensions and to treat it as a visual constraint in VRHE. However, this last approach needs more computational time. The Figure 8 represents the position estimation according to $u$ (left column) and $v$ axis (right column) for one case. We clearly see the instants when the person has been hidden by the obstacle. During these events, the position estimation according to $v$ axis does not follow the measure which is erroneous. Indeed, a person 
could not have moved so rapidly.
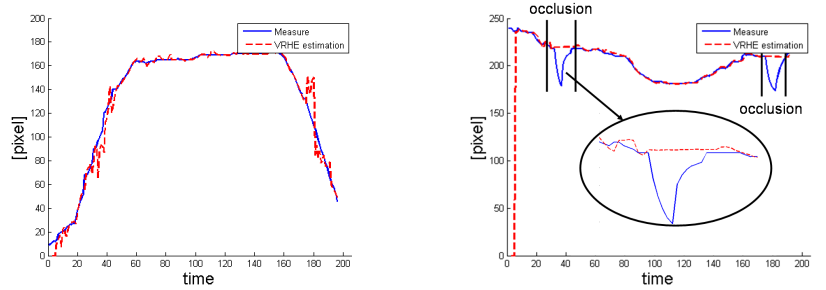

Fig. 8. Time history of the measured trajectory and the estimated trajectory

The feasibility of the approach has been tested with the estimation of position of feet. It can also be applied to the position of the middle of the box's top side corresponding to the head position. Residual generation, for both bottom and top positions, can be used in order to discriminate a fall from an occlusion and to generate an alert signal (Figure 9). The Table I illustrates the different decisions depending on the value of the residual linked to the top position of the box $r t$ and the value of the residual linked to the bottom position $r b$. If $r t=1$ and $r b=0$, the system looks with attention what will happen and if the situation stays the same, an alert will be set off. This constitutes a first step of fall detection diagnosis. Indeed we do not yet consider all the possible fall configurations.

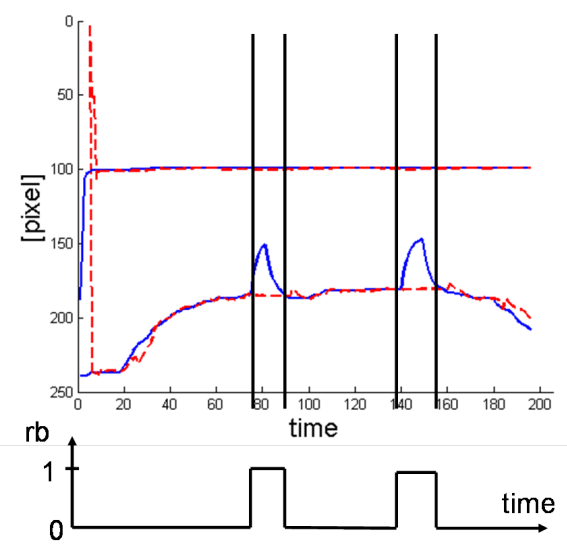

Fig. 9. Representation of top and bottom trajectories according to v. Residual generation $\mathrm{rb}$

\begin{tabular}{|c|c|l|}
\hline rt & rb & Decision \\
\hline \hline 0 & 0 & no alert \\
\hline 0 & 1 & occlusion if there is a visual constraint, noise otherwise \\
\hline 1 & 0 & caution. If the situation lasts, alert \\
\hline
\end{tabular}

TABLE I

DIAGNOSTIC OF RESIDUAL GENERATION.

\section{CONCLUSION}

A method, based on the RHE principle, for a position estimation of a moving object and its application to fall detection has been proposed in this paper. The estimation task is formulated into an optimization problem in the image plane over a past horizon. It can also deal with constraints as occlusion $\mathrm{s}$ or displacement limitations. A global model combining the camera model and the nonholonomic human motion model has been defined. The experimental results confirm the feasibility and the accuracy of the proposed approach. For both feet and head positions, residuals are generated and are used to detect falls. Other complex fall configurations are under investigation.

\section{ACKNOWLEDGEMENT}

We especially thank all our partners involved in the CAPTHOM project. This work was realized with the financial help of the French Industry Ministry and local collectivities, within the framework of the CAPTHOM project of the Competitiveness Pole $S^{2} E^{2}$, www.s2e2.fr.

\section{REFERENCES}

[1] M. Chan, D. Estève, C. Escriba and E. Campo, A review of smart homes-Present state and future challenges. Computer Methods and Programs in Biomedicine, Volume 91, Issue 1, pp. 55-81, 2008.

[2] I. Masri, T. Boudet and A. Guillot, Hyperfrequency detection method and detector using said method, patent nb. EP1818684, August 2007.

[3] C.F. Tsai and M.S. Young, Pyroelectric infrared sensor-based thermometer for monitoring indoor objects, Review of Scientific Instruments 74 (12), pp. 5267-5273, 2003,doi:10.1063/1.1626005.

[4] Y. Benezeth, B. Emile, H. Laurent and C. Rosenberger, A Real Time Human Detection System Based on Far Infrared Vision, ICISP 2008, Elmoataz et al. (Eds.), LNCS 5099, Springer, pp. 76-84, 2008.

[5] B. Schiele, M. Andriluka, N. Majer, S. Roth and Christian WojekVisual, People Detection Ú Different Models, Comparison and Discussion. In Proceedings of the IEEE ICRA 2009 Workshop on People Detection and Tracking, Kobe (Japan), 2009.

[6] P. Viola, M. Jones, and D. Snow, Detecting pedestrians using patterns of motion and appearance. In Proceedings of the Ninth IEEE International Conference on Computer Vision (ICCV 2003), 2003.

[7] S. Lee and Y. Kay, An accurate estimation of 3-D position and orientation of a moving object for robot stereo vision: Kalman filter approach, proceedings of 1990 IEEE international conference on robotics and automation, pp. 414-419, 1990.

[8] J. Wang and W.J. Wilson, 3D relative position and orientation estimation using Kalman filter for robot control, proceedings of 1992 IEEE international conference on robotics and automation, pp. 2638-2645, 1992.

[9] B. Leibe, K. Schindler and L. Van Gool, Coupled Detection and Trajectory Estimation for Multi-Object Tracking, ICCV 2007. IEEE 11th International Conference on Computer Vision, Issue, 14-21, pp. $1-8$.

[10] R. Cucchiara, A. Prati, and R. Vezzani, A multi-camera vision system for fall detection and alarm generation, Expert Systems Journal, vol. 24, no. 5, pp. 334-345, 2007.

[11] V. Lippiello, B. Siciliano and L. Villani, Visual motion estimation of $3 D$ objects: an adaptive extended Kalman filter approach, Proceedings of 2004 IEEE/RSJ International Conference on Intelligent Robots and Systems, Volume 1, pp. 957-962, 2004.

[12] E.L. Haseltine and J.B. Rawlings, Critical Evaluation of Extended Kalman Filtering and Moving-Horizon Estimation, Industrial and Engineering Chemistry Research, 44 (8), pp. 2451-2460, 2005.

[13] C.V. Rao, J.B. Rawlings and D.Q. Mayne, Constrained state estimation for nonlinear discrete-time systems: Stability and moving horizon approximations, IEEE Trans. Auto. Cont., 48(2):246-258, February 2003.

[14] E. Pastor et al., Computing the rate of spread of linear flame fronts by thermal image processing, Fire safety Journal 41, pp. 569-579, 2006.

[15] G. Arechavaleta, J.P. Laumond, H. Hicheur and A. Berthoz, The nonholonomic nature of human locomotion : a modeling study. In 1st IEEE/RAS-EMBS Int. Conf. on Biomedical Robotics and Biomechatronics. Pisa, Italy, 2006. 